\title{
LYOTROPIC MESOPHASES AS DEFECTS IN BINARY MIXTURES
}

\section{O. PARODI}

\author{
Centre de Dynamique des Phases Condensées $\left({ }^{*}\right)$, Laboratoire de Minéralogie, U.S.T.L., \\ Place Eugène-Bataillon, 34060 Montpellier Cedex, France
}

(Reçu le 12 juillet 1976, accepté le 30 juillet 1976)

\begin{abstract}
Résumé. - La classification des défauts dans les milieux ordonnés est appliquée au cas des mélanges binaires. Les défauts stables doivent être des surfaces, qui sont dans ce cas les interfaces. Pour les lyotropes, une énergie d'interface négative conduit à une valeur maximale de l'aire interfaciale. On obtient ainsi, suivant la concentration en surfactant, des micelles, puis des phases cristaux liquides qui doivent être considérées comme des phases ordonnées de défauts analogues aux réseaux de vortex dans les supraconducteurs de type II.
\end{abstract}

Abstract. - The classification of defects in ordered media is applied to the case of binary mixtures. Stable defects must be surfaces, and, in that case, interfaces. For lyotropic systems, a negative surface energy leads to a maximization of the interface area. One thus obtains, with increasing surfactant concentration, micelles, and then liquid crystal phases which must be considered as ordered defect phases, similar to vortex lattices in type II superconductors.

1. Introduction. - In a recent paper [1], Toulouse and Kleman discussed the stability of defects as determined i) by the space dimensionality, $d$, and ii) by the dimensionality of the order parameter, $n$. Their main result was that if the order parameter is an $n$-component vector, the dimensionality of topologically stable defects is

$$
d^{\prime}=d-n \text {. }
$$

In this paper, this result is applied to the case of binary mixtures, with $d=3, n=1$, which consequently implies $d^{\prime}=2$. This means that stable defects in binary mixtures are surfaces.

This concept is then applied to the special case where the binary system is made of water and an amphiphilic material, which is the case of lyotropic systems. Considerable work has been published on the thermodynamics of lyotropic system, and especially on that of micelles [2].

A first approach, refered to as the mass action law approach [3-6] considered the formation of micelles as similar to a polymerisation which spontaneously stops for a maximum aggregation number $N$ :

$$
A_{p-1}+A \rightarrow A_{p} \quad p \leqslant N
$$

with a step-wise association constant $K_{p}$ constant over the range $3 \leqslant p \leqslant N$. A convenient ratio $K_{p} / K_{2}$

$\left.{ }^{*}\right)$ Laboratoire associé au C.N.R.S. explains the observed quasi-monodispersitivity. But this approach does not explain why the aggregation process is suddenly stopped for $p=N$. A more recent version [7]. which does not use this restriction, explains the formation of larger micelles, observed at higher concentrations, but fails to explain the existence of spherical micelles observed (in the same systems) over a large concentration range near the critical micellar concentration (c. m. c.).

Another approach has also been used, where micelles are considered as thermodynamic phases [8]. The puzzling point here is that, in a thermodynamic phase, volume is an extensive quantity, which it is not for the case for micelles : if, in a micellar solution, and except for low water concentrations, one changes the total water concentration, one changes the number of micelles, but not the volume of each of them.

In fact, this strange behaviour of micelles is very similar to that observed in type II superconductors : when the magnetic field is increasing from $H_{\mathrm{c} 1}$ to $H_{\mathrm{c} 2}$, the number of vortices increases, but each vortex keeps the same characteristics.

2. Order parameter in binary mixtures. - Consider a binary system of two compounds, say A and B, and let $C$ be the concentration in compound A. At high temperature, if one neglects fluctuations, $C$ is uniform over the whole solution. If one now cools the solution below the demixion temperature, $T_{\mathrm{c}}$, two phases appear with concentrations $C_{\mathrm{I}}(T)$ and $C_{\mathrm{II}}(T)$ (Fig. 1).

From a phase transition point of view, the high 
temperature phase is the disordered phase, the low temperature one being ordered. It must here be emphasized that this low temperature phase is one thermodynamic phase with two internal states, I and II. The order parameter there has amplitude

$$
|\eta|=C_{\mathrm{I}}-C_{\mathrm{II}}
$$

with phase +1 in the internal state $I$ and phase -1 in the internal state II.

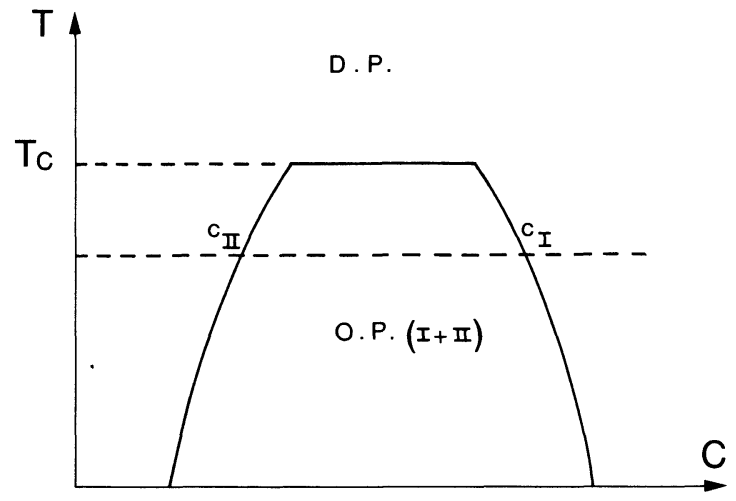

FIG. 1. - For $T<T_{\mathrm{c}}$ and $C_{\mathrm{I}}<C<C_{\mathrm{II}}$ one has the ordered phase, with a non-zero order parameter and coexistence of domains I and II with order parameter phase \pm 1 (the usual phase seggregation).

One therefore arrives at a picture of domains (usually called phases) with internal states I and II separated by interfaces, just as domains in a ferromagnetic material are separated by Bloch walls. However, one must point out a fundamental difference between interfaces and Bloch walls.

In ferromagnetic materials, the order parameter is a vector (the magnetization) and its phase can undergo a continuous variation from one domain to another. This is the case of Bloch walls, and, as a consequence, Bloch walls cannot be considered as defects. On the contrary, in the case of interfaces in binary mixtures, the phase of the order parameter is quantized, and, as a consequence, through an interface, the amplitude of the order parameter must decrease to zero, and then resume its original value. In that sense interfaces are defects and they are the stable two-dimensional defects predicted by the Toulouse-Kleman theory.

In systems like type II supraconductors, helium or nematics, the order-parameter phase is continuous and this imposes some quantization properties : quantization of flux, vorticity or disorientation. In the case of interfaces, the only requirement is that interfaces separate two domains with opposite phases, i.e. that they are surfaces separating the sample into two connected parts.

3. Application to lyotropic phases. - For most binary mixtures, the surface free-energy is positive. As a consequence the interface will have, at equilibrium, a minimum area. As an example, droplets of oil in water tend to aggregate, and the final equilibrium configuration has only two domains, one in internal state I (water) and the other in internal state II (oil).
This is not the case for amphiphilic systems. For an amphiphilic molecule, the free-energy is lowered if its polar head is in contact with water (formation of hydrogen bonds, or electrostatic interaction with counter-ions solved in water) and if its paraffinic tail is included in a paraffinic medium (hydrophobic interaction), that is if this molecule is in an interface position. In other words, this means that the interface free-energy is negative for $T_{\mathrm{k}}<T<T_{\mathrm{c}}$ (where $T_{\mathrm{k}}$ is the Krafft-plateau temperature) [9]. In that case, in the ordered phase, the total interface area will tend to be a maximum, with the restrictions due i) to the connectivity properties and ii) to the amphiphilic character of the interface.

Consider now a phase-diagram (Fig. 2) and a decrease in the water concentration $C$. For $C>C_{1}$, we are in the disordered phase. If $C$ decreases below $C_{\mathrm{I}}$, we arrive in the ordered phase with coexistence of domains I and II. The surface energy is maximum for a maximum surface/volume ratio for state II, which is achieved for spheres of minimum radius, i.e. for a radius of the order of the molecular length. This is exactly the description of micelles.

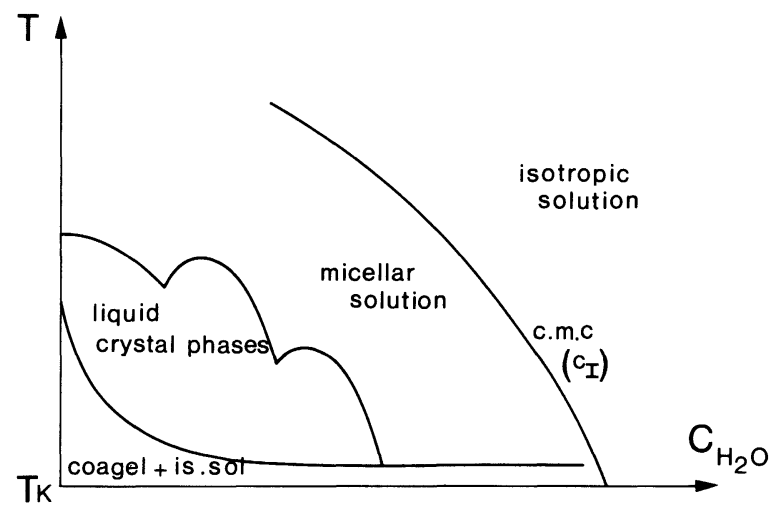

Fig. 2. - A typical phase diagram for lyotropic systems (the concentration axis is not to scale).

If $C$ is further decreased, the number of micelles increases and their interaction can no longer be neglected. This interaction is repulsive for two reasons : i) a water layer is needed between two interfaces and ii) for ionic micelles, the Coulomb interaction is repulsive. There must therefore exist some characteristic length $\lambda$, for this interaction, and a critical density of micelles $N \sim \lambda^{-3}$. Below the corresponding water concentration, one cannot form new micelles, and, as a result, the increase in the total volume of state II is generally achievea through a deformation of the micelles which take an ellipsoïdal shape (there are some special cases where this deformation process does not occur).

A futher decrease in the water concentration will cause an aggregation of micelles. The situation is now rather complex and depends on the exact form of the interaction of micelles, and probably also on the surface curvature terms in the surface energy. Roughly, two cases can arise. 
a) LIQUID CRYSTAL MESOPHASES. - In this case micelles aggregate in rods forming an hexagonal lattice. The surfaces are now cylindars, which give a lower $S / V$ ratio for state II. This hexagonal lattice is quite analogous to the one formed by vortices in type-II superconductors (or liquid helium) when the magnetic field (or vorticity) is increased. A new decrease in the water concentration leads to the well-known phase transitions in lyotropic mesophases which now appear like phase transitions in the defect lattice enabling an increased penetration of state II (surfactant) in state I (water) [10]. The great variety of phase diagrams reflects the number of parameters influencing the exact form of the surface free-energy (nature of polar heads, of counterions, length and ramification of aliphatic chains, etc...)

b) AMORPHOUS GELS. - The other case is that where the aggregation of micelles results in amorphous clusters. In this case, there is no phase transition : an increase in the surfactant concentration induces a percolation process leading to an amorphous flocculating gel (which is of a quite different nature from the low-temperature coagels and gels characterized as ordered phases).

A further increase in surfactant concentration leads to formation of inverse micelles, which correspond to the penetration of state I (water) in state II (surfactant).

Here the defects are again spheres. The curvature terms in the surface energy must play a central role in the determination of the micelle radius.

4. Discussion and conclusion. - In recent years, a controversy concerning the formation of micelles has opposed two approaches : the mass action law and the phase segregation approaches. The present approach is not to be identified with the phase segregation approach.

It must emphasized that the demixion transition is of first order ( $\eta$ and $-\eta$ do not refer to the same physical situation). Then the micelle formation must occur through a nucleation process, which is in agreement with the role of impurities which seem to favorize the formation of micelles. It can be reasonably thought that this nucleation process involves a set of equilibrium reactions between preaggregates and monomers, and that reaction rates are essentially governed by diffusion processes, as was recently found by Aniansson et al. [6]. Conductivity and diffusion of marked molecules [11] seem to prove the presence of a notable concentration of preaggregates near the c.m.c. [2]. In spite of the fact that the hydrophobichydrophilic interaction is responsible for the existence of preaggregates, small preaggregates must be considered as very different from micelles in the sense that their surface, as far as one can speak of it, is completely homogeneous. They can however form germs for the nucleation of micelles.

The present approach explains also the solubilization properties of micelles. Here also the curvature terms in the surface free-energy must play an important role. The well known effect of cosurfactants is probably due to some modification in these curvature terms.

It also solves the apparent paradox of order in lyotropic liquid crystal phases : the coexistence of a short-range disorder and a long-range order. These mesophases appear like intermediate phases with ordered defects, and are quite analogous to the intermediate state in type-II superconductors with ordered vortices.

Acknowledgments. - I am greatly indebted to Dr G. Toulouse who drew my attention on the analogy between micellar solutions and type II superconductors.

I also want to thank here Pr P. Delord and Drs B. Lindman, N. Kamenka, J. Rouvière and A. E. Skoulios for many fruitful discussions.

\section{References}

[1] Toulouse, G. and Kleman, M., J. Physique Lett. 37 (1976) L-149.

[2] For a recent review paper, see e.g. Ekwall, P. and Stenius, P. Aggregation in Surfactant systems in M.T.P. International Review of Sciences, Phys. Chem. Sci. 2. vol. 7; Surface chemistry and Colloids, M. Kerker ed. (1974).

[3] Graber, E. and Zana, R., Kolloid-Z. Z. Polym. 238 (1970) 479.

[4] Krescheck, G. C., Hamori, E., DavenPort, G. and Sheraga, H. A., J. Am. Chem. Soc. 88 (1966) 246.

[5] Muller, N., J. Phys. Chem. 76 (1972) 3017.

[6] Anianson, E. A. G., Wall, S. N., Almgren, M., Hoffmann, H., Kielmann, I., Ulbricht, W., Zana, R., Lang, J. and Tondre, C. J. Phys. Chem. 80 (1976) 905.

[7] Mukerjee, P., J. Phys. Chem. 76 (1972) 565.
[8] Hall, D. G., Kolloid. Z. Z. Polym. 250 (1972) 895.

[9] See e.g. Landau, L. et LIFCHITz, E., Physique statistique (Editions Mir, Moscou) 1957, p. 549. The authors argue that a negative surface energy would lead to a complete dissolution. This is not true in the case of amphilic system where only a part of the surfactant molecule (polar head) contributes to the surface, the other part being in bulk.

[10] Note that cylindars or double plans have good connexity properties.

[11] Lindman, B. and Brun, B. J. Colloid Interface Sci. 42 (1973) 388 ;

Lindman, B., KamenKa, N. and Brun, B. Biochim. Biophys. Acta 285 (1972) 118 